\title{
Fingerprint pattern recognition from bifurcations: An alternative approach
}

\author{
A. Castañeda-Miranda ${ }^{1,2}$, R. Castañeda-Miranda ${ }^{3}$, V.M. Castaño ${ }^{1} *$ \\ ${ }^{1}$ Centro de Física Aplicada y Tecnología Avanzada Universidad Nacional Autónoma de México Boulevard Juriquilla 3001 Santiago \\ de Querétaro Querétaro, 76230, México \\ ${ }^{2}$ Escuela Superior de Apan, Universidad Autónoma del Estado de Hidalgo, Calle Ejido De Chimalpa Tlalayote S/N, Col. Chimalpa, \\ Apan, Hidalgo, 76010, México \\ ${ }^{3}$ Unidad Académica de Ingeniería Eléctrica Universidad Autónoma de Zacatecas, Zacatecas, Zacatecas, 98000, México \\ *Corresponding author E-mail: meneses@unam.mx
}

Copyright $\odot 2015$ V.M. Castaño et al. This is an open access article distributed under the Creative Commons Attribution License, which permits unrestricted use, distribution, and reproduction in any medium, provided the original work is properly cited.

\begin{abstract}
A pc-based automatic system for fingerprints recording and classification is described, based on the vector analysis of bifurcations. The system consists of a six-step process: a) acquisition, b) preprocessing, c) fragmentation, d) representation, e) description, and f) recognition. Details of each stage, along with actual examples of fingerprints recognition are provided.
\end{abstract}

Keywords: Fingerprints; Dactiloscopy; Pattern Recognition; Pc-Based Software; Segmentation.

\section{Introduction}

It is well known, that fingerprints recognition and classification allow the unique identification of a person, since his/her fingerprint presents natural patterns in an almost infinite variety of combinations. Even in the case of highly similar fingerprints, these combinations are so unique as to prevent two exactly identical fingerprints as it indicated in different biography as solved elsewhere [1], [2], [3], [4], [5], [6]. The practical applications of fingerprints recognition and characterization ranges from public or private safety, to forensic medicine, legal matters, among other applications, a review of it with a statistical analysis was done by Abraham et al. as solved [5]. In fact, the recognition and classification of fingerprints, either from their minutae or their mutual mathematical correlation, represents a very active area of R\&D nowadays and a number of methodologies have been proposed, including hybrid matchers, e.g., as solved by Ross et al. as solved [7] and wavelet transforms, e.g., as solved by Nanni et al. as solved [8]. From the Forensic Science point of view, there exist two main methods for fingerprints identification, based on a standard, and somewhat arbitrary, classification. Indeed, whereas in South America and parts of Europe the Vucetich method is employed, in United States and England the Henry classification scheme is employed.

From the pattern recognition technology standpoint as solved elsewhere [9], [10], either classification is made by surveying the fingerprint, thus assigning a denomination, according to the particular scheme utilized, that will identify the given finger. In this way, by using the ten fingers of the hand, one will have a combination that will identify uniquely a person. It can happen, however, that the combination is repeated and therefore a sub-classification must be devised. This is based on the topological characteristic of the patterns of the fingerprints, such as branching, merging, islands, etc. Nevertheless, this process requires the meticulous examination of experts in dactiloscopy, which turns the classification of fingerprints into a cumbersome and slow process that relies on the skill, mood and honesty of the expert making the identification. 


\section{Objectives}

Accordingly, this work is aimed to describing a pc-based automatic system for digitizing and analyzing fingerprints, by extracting the topologically-essential points that allow to distinguish one fingerprint from another, based on a simple vector análisis of the bifurcations in the fingerprints. These peculiarities within the fingerprint are known as characteristic points, and can be classified into interruptions in the combs, abrupt end, ramifications and fusions. The characteristic points and their relative position are of key importance for the effective the comparison of two fingerprints, and though each fingerprint contains about 100 characteristic points, it is usually enough to analyze a dozen to positively identify a standard.

\section{Methods}

Fingerprint analysis are usually performed methods using laboratories. However, a case here exists. In the event, that there is to collect information less than $10 \%$ of the fingerprint, the process requires a rebuilding process for identification, such as to reduce backlogs, but as a result of this information also, provides a variety of information and verify results, which harms directly results or handle high-profile cases. The following section provides a method of fingerprint identification using at least $10 \%$ of the dactilar footprint for reconstruction and is based on a Euclidean distance with its closest neighbors is presented.

\subsection{Block diagram of the system}

The whole system consists of six stages, schematically shown in the diagram of Figure 1 . As can be observed there, the system itself is extremely simple and modular, allowing to update and improve the different stages quite easily.

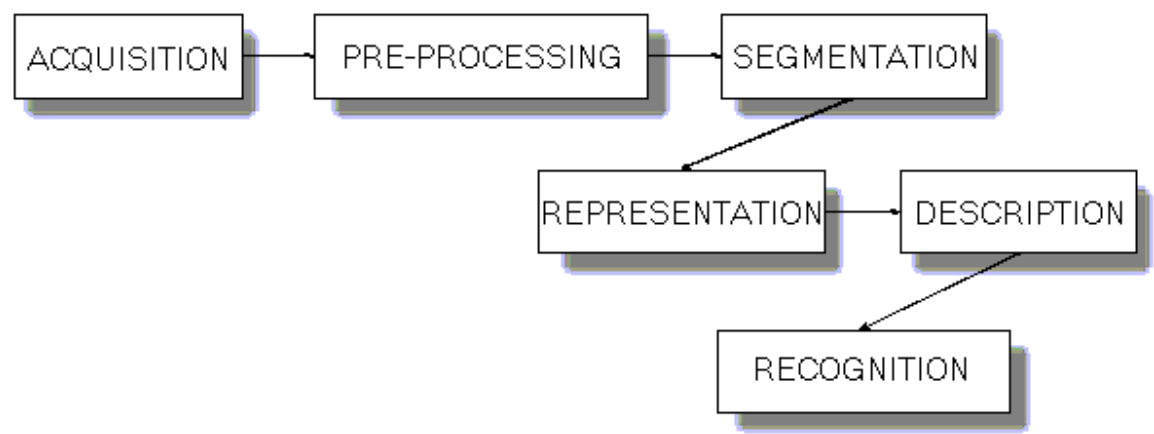

Fig. 1: Stages of the System for Fingerprints Recognition

\section{Results}

For the description process, a tracking of the fingerprint around the point's interest is performed, analyzing each pixel in relation with two or more of its neighbors. Depending on its vicinity, each pixel belongs to one of the three following cases:

1) The basic type of forking.

2) A step line.

3) A square line.

The first case is so due to the fact, that a forking requires at least of two pixels to represent two ramifications. The second and third case tell us that, the fact of having two pixels do not ensure that a forking will be found, since there exist lines that cannot be made sharper, digitally speaking, without facing the risk of losing connection. Figure 2 shows schematically the three above cases.

To distinguish among these three cases, their corresponding neighbors must be carefully analyzed to characterize each one. As can be observed in figure 2, one can distinguish a forking from an echeloned line or a square, through the analysis of each pixel. In the case of these three types having the same amount of neighbors, the difference is found in the position that they may have with respect to the given pixel. Some connectivity criteria must also, be applied: if the pixel has four neighbors, it can be likely assigned to forke condition. 


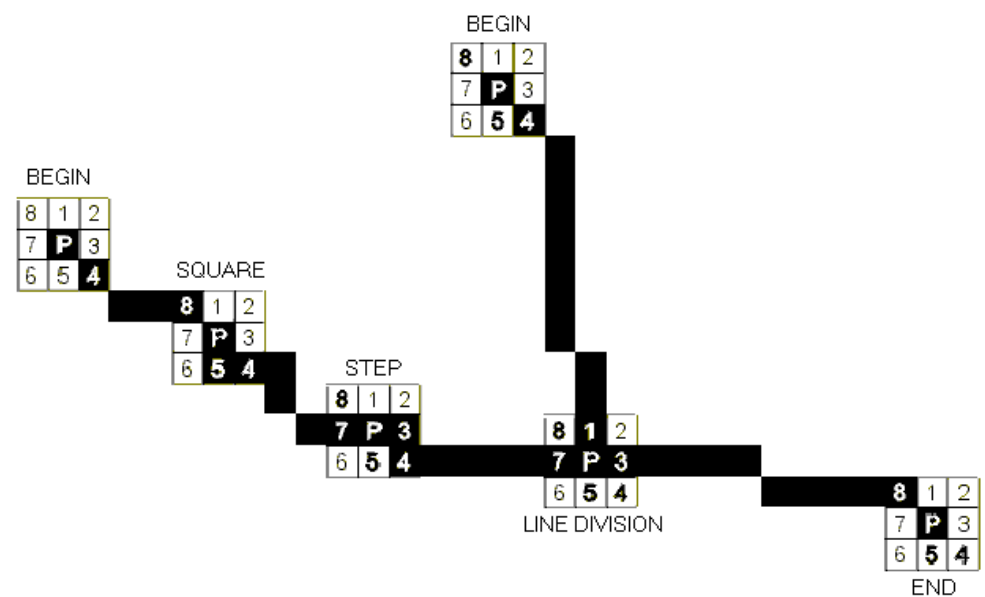

Fig. 2: Diagram Representing the Cases of Two Neighbors within a Region.

The characteristic points play an important role in the identification of a fingerprint, being the principal characteristic taken into account in this work, the forking of the combs. For obtaining the forking within the fingerprint a tracking algorithm (bifur.exe), programmed in $\mathrm{C}$, is employed. This algorithm provides the coordinates of all the forkings found within the fingerprint. A typical set of coordinates is shown in Table 1.

Table 1: Coordinate Data of the Forking Found by the Tracking Algorithm.

\begin{tabular}{llllllllllll}
\multicolumn{10}{c}{ Table 1: Coordinate Data of the Forking Found by the Tracking Algorithm. } \\
\hline No & $\mathrm{x}$ & $\mathrm{y}$ & No & $\mathrm{X}$ & $\mathrm{y}$ & No & $\mathrm{x}$ & $\mathrm{y}$ & No & $\mathrm{x}$ & $\mathrm{y}$ \\
\hline 1 & 49 & 25 & 16 & 230 & 112 & 31 & 209 & 195 & 46 & 195 & 277 \\
2 & 18 & 30 & 17 & 168 & 121 & 32 & 364 & 198 & 47 & 24 & 287 \\
3 & 203 & 31 & 18 & 466 & 123 & 33 & 4 & 209 & 48 & 152 & 288 \\
4 & 295 & 49 & 19 & 147 & 128 & 34 & 134 & 210 & 49 & 194 & 297 \\
5 & 326 & 68 & 20 & 124 & 142 & 35 & 87 & 212 & 50 & 82 & 310 \\
6 & 50 & 71 & 21 & 179 & 158 & 36 & 29 & 216 & 51 & 130 & 317 \\
7 & 89 & 85 & 22 & 363 & 160 & 37 & 191 & 216 & 52 & 163 & 318 \\
8 & 110 & 88 & 23 & 262 & 163 & 38 & 96 & 219 & 53 & 130 & 321 \\
9 & 141 & 91 & 24 & 248 & 167 & 39 & 73 & 227 & 54 & 220 & 328 \\
10 & 364 & 93 & 25 & 214 & 171 & 40 & 137 & 230 & 55 & 68 & 332 \\
11 & 141 & 95 & 26 & 476 & 171 & 41 & 58 & 252 & 56 & 265 & 337 \\
12 & 148 & 96 & 27 & 102 & 179 & 42 & 185 & 254 & 57 & 392 & 345 \\
13 & 17 & 107 & 28 & 484 & 183 & 43 & 82 & 255 & 58 & 268 & 354 \\
14 & 142 & 111 & 29 & 123 & 184 & 44 & 246 & 263 & 59 & 235 & 360 \\
15 & 213 & 111 & 30 & 43 & 190 & 45 & 449 & 266 & & \\
\hline
\end{tabular}

Once the tracking of the characteristic points is carried out and the forking characteristics of the fingerprint are identified (figure 3), coordinates are assigned, represented in the equation 1.

$$
H(p)=\left\{\begin{array}{c}
p_{1}\left(x_{1}, y_{1}\right) \\
p_{2}\left(x_{2}, y_{2}\right) \\
p_{3}\left(x_{3}, y\right) \\
\vdots \\
p_{n}\left(x_{n}, y_{n}\right)
\end{array}\right\}
$$

Where $\mathrm{H}(\mathrm{p})$ is the set that identifies the fingerprint, being Pi(xi,yi) a set of points and xi, yi being the horizontal and vertical coordinates of the point $i$, respectively. 


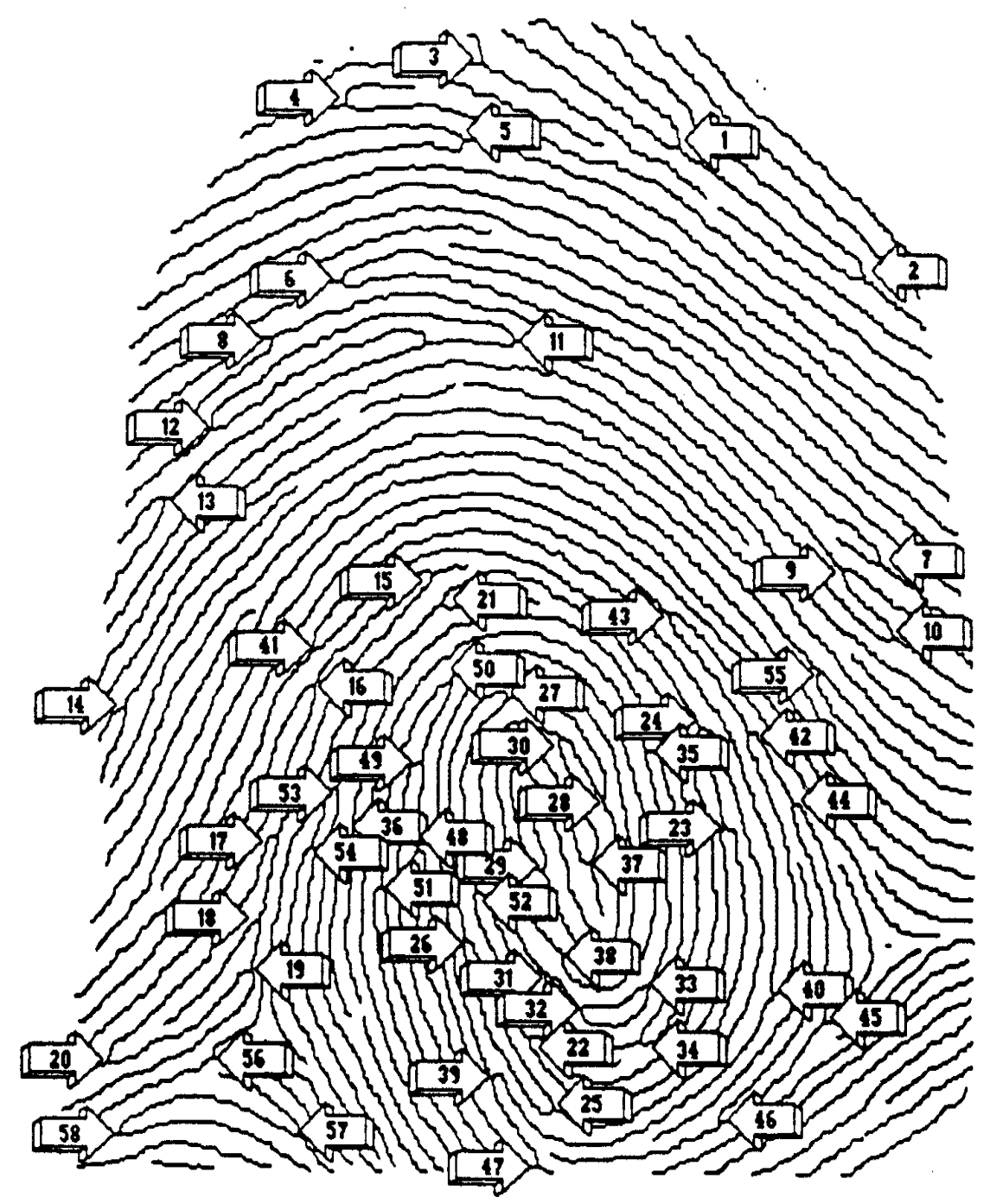

Fig. 3: Image Form with Their Detected Forking.

Next, once the position of the first point $\mathrm{P} 1(\mathrm{x} 1, \mathrm{y} 1)$ in the fingerprint is established, the nearest five points are located, as is observed in figure 4 .

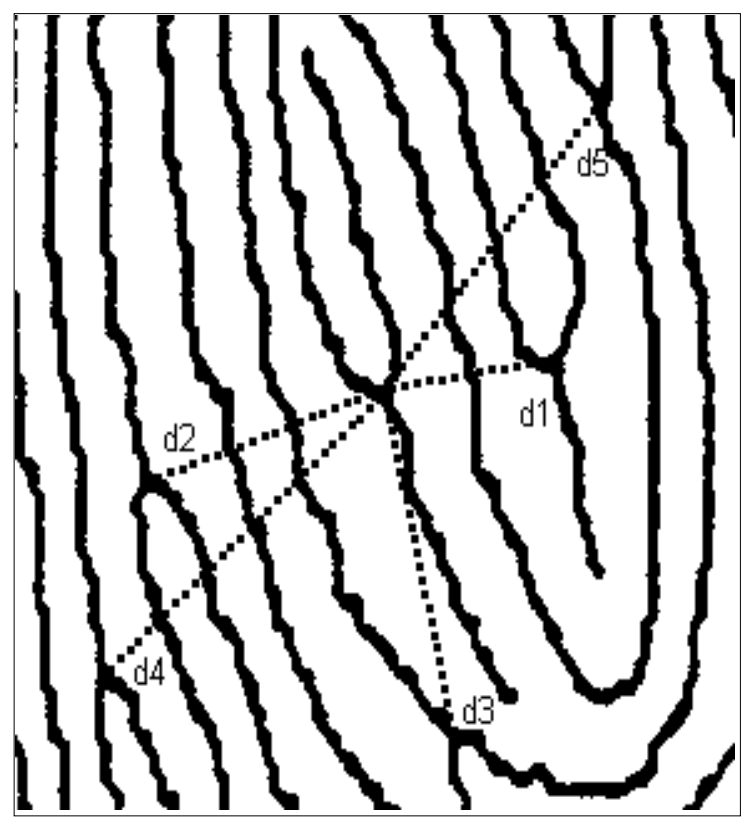

Fig. 4: Image of the Relationship of Distances between the Characteristic Points 
Based on the Euclidian distance between points, the values of the five distances to a group that will be identify to the point p1 are assigned. This group of distances defines the standard vector that determines t the first point (i.e p1) and will be identified as D1.

By reproducing this process for each point of $\mathrm{H}(\mathrm{p})$ we will obtain a standard vector for each one. As a rule for each point $\mathrm{i}$, the corresponding vector is represented for equation 2 .

$D_{i}\left[p_{i}\left(x_{i}, y_{i}\right)\right]=\left\{\begin{array}{l}d_{1} \\ d_{2} \\ d_{3} \\ d_{4} \\ d_{5}\end{array}\right\}$

Where $\mathrm{d} 1, \mathrm{~d} 2, \mathrm{~d} 3, \mathrm{~d} 4$ and $\mathrm{d} 5$ are the minimal distances from the Pi position (xi, yi) towards the five nearest points. The distances are ordered so that $\mathrm{d} 1<\mathrm{d} 2<\mathrm{d} 3<\mathrm{d} 4<\mathrm{d} 5$.

Once the five vectors are defined, their total length in 5 dimensional space is calculated, according to the equation 3 :

$$
L\left[p_{i}\left(x_{i}, y_{i}\right)\right]=\left\{\sum_{j=1}^{5}\left(d_{j}\right)^{2}\right\}^{1 / 2}
$$

\section{Conclusions}

Effective identification of fingerprints can be achieved by means of a pc, provided the proper software is utilized. The topological characteristics of each fingerprint are key for a successful identification, therefore it is recommended the use an optical system with enough resolution for the acquisition of the fingerprint, instead of digitizing a printout. The vector technique provides a useful tool to make invariable the system to adjournments of the images of the fingerprints. The test performed on our system show that it constitutes a reliable tool and some work towards addition of some mathematical morphogenesis concepts, is under way.

\section{Acknowledgements}

The authors wish to thank Rene Preza-Cortés, Carmen Vázquez and Adrián Oskam for their technical support. The financial support of CONCYTEQ (QRO-2005-C01-15218) is also, gratefully acknowledged. '

\section{References}

[1] M. Sayed, Coset decomposition method for storing and decoding fingerprint data, Journal of Advanced Computer Science \& Technology, vol. 4, num. 1, pp 6-11, (2015)

[2] A. Jain and S. Aggarwal, "Multimodal Biometric System: A Survey", International Journal of Applied Science and Advance Technology, vol. 1 , no. 1, pp. 58-63, 2012.

[3] T.H. Nguyen, Y. Wang, R. Li, An improved ridge features extraction algorithm for distorted fingerprints matching, Journal of Information Security and Applications, vol. 18, num. 4, pp. 206-214, 2013. http://dx.doi.org/10.1016/j.jisa.2013.11.001.

[4] D. Maltoni, D. Maio, A.K. Jain, S. Prabhaker, Handbook of Fingerprint Recognition, Springer-Verlag, London, 2009. http://dx.doi.org/10.1007/978-1-84882-254-2.

[5] J. Abraham, C. Champod, C. Lennard, C. Roux, Modern statistical models for forensic fingerprint examinations: A critical review, Forensic Science International, vol. 232, num. 1-3, pp. 131-150, 2013. http://dx.doi.org/10.1016/j.forsciint.2013.07.005.

[6] J.A. Speir, J. Hietpas, and Frequency filtering to suppress background noise in fingerprint evidence: Quantifying the fidelity of digitally enhanced fingerprint images, Forensic Science International, Vol. 242, p94-102, 2014. http://dx.doi.org/10.1016/j.forsciint.2014.06.026.

[7] A. Ross, A. Jain, J. Reismanb, A hybrid fingerprint matcher, Pattern Recognition, vol. 36, pp 1661- 1663, 2003. http://dx.doi.org/10.1016/S0031-3203(02)00349-7.

[8] L. Nanni, A. Lumini, A hybrid wavelet based fingerprint matcher, Pattern Recognition, vol. 40, pp. 3146-3151, 2007. http://dx.doi.org/10.1016/j.patcog.2007.02.018.

[9] C. Neumann, C. Champod, M. Yoo, T. Genessay, G. Langenburg, Quantifying the weight of fingerprint evidence through the spatial relationship, directions and types of minutiae observed on fingermarks, Forensic Science International, Vol. 248, pp. 154-171, 2015. http://dx.doi.org/10.1016/j.forsciint.2015.01.007.

[10] H.C.Lee, R.E. Gaensslen, Advances in Fringerprint Technology, CRC Press,NewYork, 2001. 